\title{
PTSD Coach around the world
}

\author{
Eric Kuhn ${ }^{1,2}$, Christianne van der Meer ${ }^{3}$, Jason E. Owen ${ }^{1}$, Julia E. Hoffman ${ }^{1}$, Richard Cash ${ }^{4}$, Pasqualina \\ Carrese $^{5}$, Miranda Olff, ${ }^{3,6}$, Anne Bakker ${ }^{3}$, Julia Schellong ${ }^{7}$, Patrick Lorenz ${ }^{7}$, Matthias Schopp ${ }^{8}$, Heinrich \\ Rau $^{9}$, Kerstin Weidner ${ }^{7}$, Filip K. Arnberg ${ }^{10,11}$, Martin Cernvall ${ }^{10}$, Thomas Iversen $^{12}$
}

${ }^{1}$ VA National Center for PTSD, VA Palo Alto Healthcare System, Menlo Park, CA, USA; ${ }^{2}$ Department of Psychiatry and Behavioral Sciences, Stanford University School of Medicine, Stanford, CA, USA; ${ }^{3}$ Academic Medical Centre, Department of Psychiatry, University of Amsterdam, Amsterdam, The Netherlands; ${ }^{4}$ Phoenix Australia, Department of Psychiatry, the University of Melbourne, Melbourne, Australia; ${ }^{5}$ Veterans Affairs Canada, Montreal, Canada; ${ }^{6}$ Arq Psychotrauma Expert Group, Diemen, The Netherlands; ${ }^{7}$ Department of Psychotherapy and Psychosomatic Medicine, Medizinische Fakultät Carl Gustav Carus, Technische Universität Dresden, Dresden, Germany; ${ }^{8}$ Department of Computer Science, Universität der Bundeswehr München, Munich, Germany; ${ }^{9}$ German Armed Forces Center for Military Mental Health (Psychotraumazentrum), Berlin, Germany; ${ }^{10}$ National Centre for Disaster Psychiatry, Department of Neuroscience, Psychiatry, Uppsala University, Uppsala, Sweden; ${ }^{11}$ Stress Research Institute, Stockholm University, Stockholm, Sweden; ${ }^{12}$ Department of Psychology, Aarhus University, Aarhus, Denmark Correspondence to: Eric Kuhn, PhD. Dissemination and Training Division, VA National Center for PTSD, 795 Willow Rd, Menlo Park, CA 94025, USA. Email: Eric.Kuhn@va.gov.

\begin{abstract}
Posttraumatic stress disorder (PTSD) is a global public health problem. Unfortunately, many individuals with PTSD do not receive professional care due to a lack of available providers, stigma about mental illness, and other concerns. Technology-based interventions, including mobile phone applications (apps) may be a viable means of surmounting such barriers and reaching and helping those in need. Given this potential, in 2011 the U.S Veterans Affairs National Center for PTSD released PTSD Coach, a mobile app intended to provide psycho-education and self-management tools for trauma survivors with PTSD symptoms. Emerging research on PTSD Coach demonstrates high user satisfaction, feasibility, and improvement in PTSD symptoms and other psychosocial outcomes. A model of openly sharing the app's source code and content has resulted in versions being created by individuals in six other countries: Australia, Canada, The Netherlands, Germany, Sweden, and Denmark. These versions are described, highlighting their significant adaptations, enhancements, and expansions to the original PTSD Coach app as well as emerging research on them. It is clear that the sharing of app source code and content has benefited this emerging PTSD Coach community, as well as the populations they are targeting. Despite this success, challenges remain especially reaching trauma survivors in areas where few or no other mental health resources exist.
\end{abstract}

Keywords: Posttraumatic stress disorder (PTSD); trauma; mobile applications (mobile apps); public health; smartphones

Received: 14 March 2018; Accepted: 03 May 2018; Published: 25 May 2018.

doi: $10.21037 /$ mhealth.2018.05.01

View this article at: http://dx.doi.org/10.21037/mhealth.2018.05.01

\section{PTSD Coach around the world}

Trauma and consequent posttraumatic stress disorder (PTSD) is a global public health problem. In fact, about $70 \%$ of the world's population will be exposed to one or more traumas in their lifetimes, with exposed individuals experiencing an average of more than four traumas (1).
Common trauma exposures include rape, war-related experiences, physical violence, life-threatening accidents, and the unexpected death of a loved one. The lifetime prevalence of PTSD is nearly four percent of the global population and is associated with significant burden (2). PTSD involves intrusive re-experiencing of the trauma, avoidance of stimuli associated with the trauma (e.g., 
memories, places), negative changes in emotions and thoughts (e.g., inability to experience positive feelings, self-blame), and hyper-arousal (e.g., hypervigilance, sleep disturbance) (3). Many trauma survivors who do not develop diagnosable PTSD nonetheless suffer from significant symptoms and impairments, considered subthreshold PTSD (4). Those with PTSD commonly experience comorbid mental and behavioral health conditions, including depression and alcohol and substance use disorders (1).

Unfortunately, a substantial proportion of trauma survivors with significant PTSD and other symptoms do not recognize these as trauma-related and problematic so therefore do not seek professional help $(5,6)$. Many of those who do realize that their posttraumatic symptoms are problematic and thus require care may encounter additional barriers to receiving help. These include not knowing where to seek care, limited financial resources, time constraints, and a lack of available qualified mental health providers (6), especially in rural areas and developing countries (7). Even when resources are available, many will not seek care due to stigma, fear of negative consequences (e.g., on career prospects), concerns about confidentiality, poor past experiences with treatment, and beliefs that treatment will not help (6). Thus, there is a tremendous unmet need for PTSD treatment that traditional care models and existing resources cannot adequately address (7).

Technology-based mental health interventions offer promise as they can overcome distance, time, provider availability, resource limitations, as well as other possible barriers to treatment (e.g., stigma-related to mental health treatment, privacy concerns), and potentially even capitalize on the desire by some trauma survivors to tackle their symptoms on their own (6). In fact, Internet delivered cognitive-behavioral therapy-based programs have been shown to be efficacious in reducing PTSD symptoms (8). Emerging research on mobile phone-based interventions for mental health conditions, including smartphone applications (apps) is also encouraging (9). Given that smartphone ownership worldwide continues to grow (10), there is an enormous potential for apps to deliver low or no cost, easily accessible self-help interventions for trauma survivors with PTSD and other trauma-related symptoms $(6,11,12)$.

Below we discuss the development of and research on the PTSD Coach mobile app $(13,14)$, an early entrant into the field of mobile mental health. We then describe versions of PTSD Coach that have been or are being developed by individuals and entities (e.g., governmental agencies, academic institutions) from other countries, highlighting the significant adaptations, advances, lessons learned, and when available emerging research on these variants. Lastly, we discuss the benefits, challenges, and promises of this model of globally disseminating a mobile app for PTSD.

\section{PTSD Coach}

The lifetime prevalence of PTSD in the U.S population is $6.8 \%$ (15). For military veteran populations who have been deployed to warzones rates of current PTSD are roughly $12-15 \%$ (16-18). At the beginning of 2018, an estimated $77 \%$ of U.S. adults owned a smartphone up from just $45 \%$ 5 years ago (19). Given this level of PTSD and increasing rate of smartphone ownership, the U.S. VA National Center for PTSD, Dissemination and Training Division developed PTSD Coach (version 1.0), which became available for free download in the App Store in March 2011 and in Google Play in June 2012. As of March 2018, PTSD Coach (now version 3.0) has been downloaded over $350 \mathrm{~K}$ times $(\sim 216 \mathrm{~K}$ for iOS, $\sim 135 \mathrm{~K}$ for Android) in 106 countries and has received awards for its accessibility for individuals with disabilities (20) and innovation (21).

In 2009, noticing an increasing trend of veterans, especially younger ones, attending VA PTSD treatment programs using smartphones, a team of clinical psychologists, who were already developing web-based interventions for trauma survivors (22) (e.g., afterdeployment.org), proposed PTSD Coach with funding from the VA National Center for PTSD. To inform the scope, features, and functions of the app, a series of focus group with veteran PTSD patients and VA PTSD providers was conducted (14). App content was drawn heavily from evidence-based, cognitivebehavioral psychotherapies for PTSD (23) and related and often comorbid conditions (e.g., behavioral activation for depression), and reliable psychoeducational materials created by the VA National Center for PTSD (www.PTSD. VA.gov).

PTSD Coach is a self-contained, psychoeducational and self-management tool for acute distress related to PTSD that targets military veterans as well as the broader civilian population. As a public health intervention, it was designed to improve mental health literacy and problem recognition to possibly motivate treatment seeking if needed by providing accurate descriptions of evidencebased treatments for PTSD, instilling hope that treatment can be helpful, and reducing stigma related to treatment seeking. PTSD Coach accomplishes these goals through 


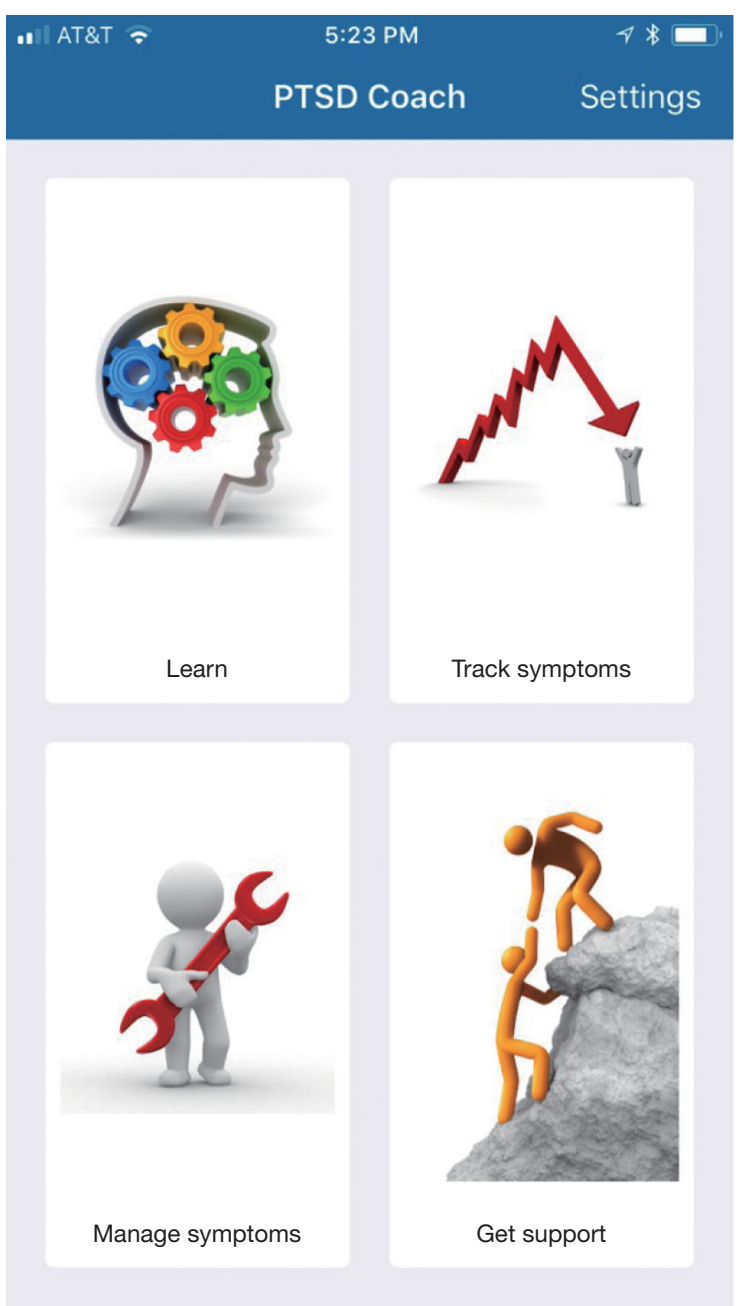

Figure 1 Home screen of U.S. PTSD Coach.

four core sections accessible through its home screen (see Figure 1). The Learn section provides psychoeducational information about PTSD (e.g., What is PTSD? How does PTSD develop?), professional care (e.g., How do I find a counselor/therapist? PTSD treatments that work), and PTSD and the family (e.g., Impact of PTSD on children). Track Symptoms allows users to complete the PTSD Checklist for DSM-5 (24) and receive interpretive feedback on their PTSD symptom severity, with recommendations for treatment if indicated. This section also offers tracking of symptoms over time with the ability to schedule reminders to periodically take the PCL-5 and view a graph of symptom change over time. The Manage Symptoms sections offers coping tools for acute distress related to PTSD symptoms. Users can select a symptom type (e.g.,
Reminded of trauma) to be offered a coping tool or select a coping tool (e.g., deep breathing), including one they may have previously created through the costume tool feature. Finally, Get Support provides easy access to crisis support resources (e.g., Veterans Crisis Line) including supportive contacts the user can add (e.g., an AA sponsor, therapist, close friend or family member).

Aside from determining the functions, features, and content of the app, the team also had to weigh important design, usability, technical, legal, and ethical considerations and requirements. Among these, as the app would be a publicly available resource, VA required that no identifiable data could be collected and transmitted from the user's mobile device. To maximize reach (e.g., to those in rural areas or on military deployments), after download, PTSD Coach could not be dependent on Internet connection for full-feature use. Likewise, to reduce memory use and allow for over the air download, the app could not include video content and only a limited number of audio files. Finally, as an electronic resource developed by a U.S. government agency, it was required (to be compliant with Section 508 of the Rehabilitation Act of 1973) that the app be accessible to those with common disabilities (e.g., visual and hearing impairments).

Regarding evaluation and research, PTSD Coach has been found to be acceptable and perceived to be moderately to very helpful for managing PTSD symptoms by veterans attending a VA residential PTSD treatment program (14). It has also shown potential in reducing PTSD symptoms among community samples after one (25) and three months of use (26), and increased app use has been shown to relate to greater PTSD symptom improvement (27). In addition, improvements in depression symptoms and psychosocial functioning have also been demonstrated (26). Furthermore, based on metrics gathered on the publicly available versions of the app, it appears that PTSD Coach is reaching individuals with elevated PTSD symptoms and is helpful for reducing acute distress (28). Finally, in a pilot study, PTSD Coach was integrated into VA primary care treatment for PTSD with clinician support and led to improved PTSD symptoms and increased receipt of specialty PTSD care following the intervention period (29). Based on these pilot findings, a two-site, full-scale VA-funded RCT is underway to test if PTSD Coach with clinician support delivered in primary care performs better than treatment as usual at reducing PTSD symptoms and engaging veterans in PTSD care. 


\section{Versions of PTSD Coach from around the world}

Given the general applicability of the information, coping tools, and assessment features in PTSD Coach, individuals from academic and governmental entities from around the world have sought to use it and tailor its content to the needs of their specific populations (e.g., language, local resources). In the spirit of "open mHealth" (30), the VA National Center for PTSD has encouraged and enabled widespread dissemination of PTSD Coach by optimizing existing code for extensibility (e.g., supporting internationalization and localization to facilitate language changes) and developing a procedure for sharing source code with collaborating nonprofit organizations. Code is shared through a web-based hosting service (i.e., GitHub.com), with requestors being given access to the file repositories they need to build their own versions of the app. VA uses common version control and code-sharing technologies to make software changes readily available to participating collaborators and to make international versions of PTSD Coach available to VA. It is also important to note that PTSD Coach source code is structured so that all app features are modular and can be readily transferred to other apps, modified, or removed. VA has pioneered methods for making tools, assessments, and learn topics readily accessible to other apps, which has made it possible to integrate tools and features that are developed for other mobile apps in VA's suite [e.g., AIMS (Anger and Irritability Management Skills), STAIR Coach (Skills Training in Affective and Interpersonal Regulation)].

In general, VA has approved code-sharing to those who share the mission to reduce the burden of PTSD by offering free, private, and accessible resources to their target audience. Recipients are asked to preserve the name, if sensible, and acknowledge that their version is based on the original PTSD Coach app. They are also encouraged to share code updates and enhancements to the app so all members of the PTSD Coach community can benefit. To date, individuals and entities from six other countries have been given the code for PTSD Coach (version 1.0) and have developed or are developing their own versions, which are described below.

\section{PTSD Coach Australia}

In Australia, the 12-month prevalence rate of PTSD is estimated to be $6.4 \%$ (31). In 2017, $88 \%$ of Australians owned a smartphone (32). In early 2012, the Australian Department of Veteran's Affairs engaged the Australian
Centre for Posttraumatic Mental Health (ACPMH, now Phoenix Australia) to develop Australian versions of PTSD Coach for the Apple and Android operating systems. These apps would specifically target PTSD amongst military personnel and veterans-who experience a 12-month PTSD prevalence rate of $8 \%$ (33). The two PTSD Coach Australia apps were launched in early 2013.

Developing the Australian versions involved several activities: revising content to suit Australian defense and veteran user groups; updating the information and assessment components to reflect developments in the PTSD literature; refreshing technical aspects of the app to suit hardware and operating system advances; and adding new app functions and capabilities. These alterations were based on advice from the U.S. development team, consultation with the Australian Defence Force and Department of Veteran's Affairs, and experience of ACPMH with developing digital resilience resources for defense and veteran populations.

In particular, revisions for PTSD Coach Australia included updating the psychoeducational content and symptom monitoring tool to be consistent with DSM-5; revising content to ensure that the language, tone, and detail were appropriate for an Australian audience including re-recording the audio information and symptom management content with two Australian voices; and adding information and contact details relating to Australian defense and veteran treatment and support services, as well as community based help-seeking pathways.

Adaptations also involved several functional enhancements and additional features. These include a 'scheduler' feature, allowing users to set reminders within the app or on their smartphone calendar to use assessment and symptom management tools, as well schedule reminders for other treatment or rehabilitation appointments or activities occurring outside the app. They also included allowing users to select symptom management tools directly - in addition to the original random presentation of tools with the option of selecting favorites of version 1.0 of PTSD Coach-as well as promoting tools that have shown benefit for the user based on previously demonstrated distress rating reductions. To accommodate defense personnel and veteran users, the app includes two separate user pathways within setup and navigation, providing targeted information about treatment and support services for these groups. PTSD Coach Australia also allows users to email results of their PTSD assessment (see Figure 2), for 


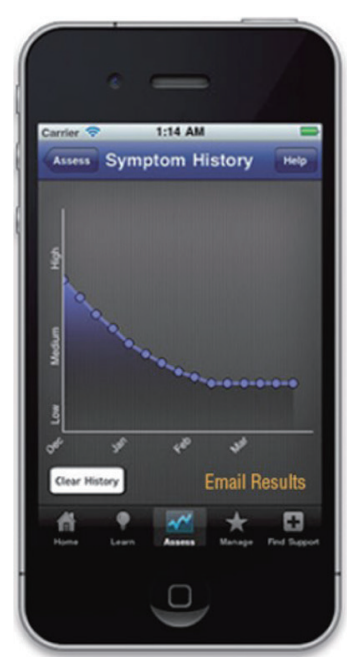

Figure 2 Symptom history screen of PTSD Coach Australia.

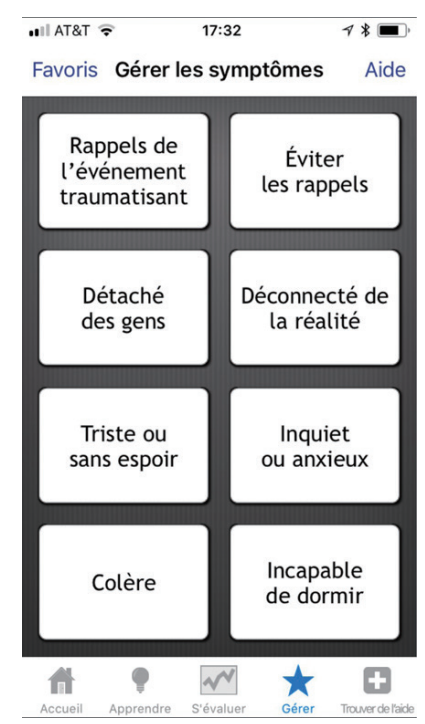

Figure 3 Manage symptoms screen of PTSD Coach Canada.

example to a healthcare professional, affording symptom monitoring data to be utilized in treatment. Additionally, a "clinician's guide" was developed to support use by mental health professionals-providing guidance for how to introduce the app to patients, use its functions, and integrate it into treatment (34).

While anecdotal feedback from healthcare providers working with Australian veterans has been very positive and app store ratings are favourable (i.e., average of 4 out of 5 stars), to date, there has been no systematic evaluation of PTSD Coach Australia.

\section{PTSD Coach Canada}

It is estimated that $8 \%$ of the Canadian population will have PTSD in their lifetime (35) and $11 \%$ of the Regular Canadian Armed Forces and reservists deployed in support of the mission in Afghanistan experience PTSD (36). As of 2016, three-quarters of Canadians owned a smartphone (37). This level of smartphone ownership and PTSD provides a significant opportunity for delivery of behavioral health interventions to Canadians. The Minister of Veterans Affairs Canada (VAC), therefore, officially launched, PTSD Coach Canada in May 2013 to assist Canadian veterans, Canadian Armed Forces members and their families, as well as the broader Canadian population impacted by PTSD (38). PTSD Coach Canada is available in both French and English (see Figure 3) via localization and can be downloaded for free from the App Store and Google Play. As of February 2018, there were over $13 \mathrm{~K}$ downloads from the App Store and more than $5 \mathrm{~K}$ downloads from Google Play.

PTSD Coach Canada is similar to the original U.S. version with several adaptations. The resource section was modified to include only mental health resources available to the targeted Canadian audiences. As well, the Learn about Professional Care section was adapted to describe how to access care within the Canadian mental health care system. The term counsellor was also replaced by the more general term mental health practitioner. Finally, information about operational stress injuries (OSI) was added to the Learn about PTSD section, which VAC defines as any persistent psychological difficulty resulting from operational duties performed while serving in the CAF or as a member of the Royal Canadian Mounted Police. This non-medical term describes a broad range of problems that include diagnosed psychiatric conditions such as anxiety disorders, depression, and PTSD, as well as other problems and conditions that may be less severe but still interfere with daily functioning (39). The hope was that the adoption of the term OSI would confer to the treatment of mental injuries the same perceived legitimacy as the treatment of physical injuries, thereby reducing stigma associated with mental health problems and care seeking (40).

To date, no systematic evaluation of PTSD Coach Canada has been conducted. Given its high correspondence with the U.S. version and the cultural similarities between these countries, the research findings on the original PTSD Coach may generalize; however, research is needed to evaluate how well the findings hold particularly for those using the app in French. Updating of the original version 


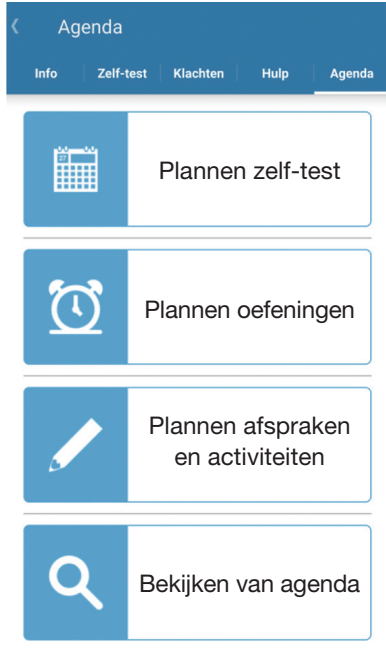

Figure 4 Agenda screen for SUPPORT Coach Netherlands.

of PTSD Coach Canada to reflect the recently released U.S version (i.e., 3.0) is in the planning stage.

\section{SUPPORT Coach Netherlands}

In the Netherlands, $80.7 \%$ of the population has experienced a potentially traumatic event (41). The lifetime prevalence of PTSD in the Dutch population is estimated to be $7.4 \%$ (41). The vast majority ( $83 \%$ in 2016 ) of the Dutch population owns a smartphone, generating great potential to deliver trauma support to Dutch speaking citizens worldwide (42). In 2015, the Psycho-trauma Team at the Academic Medical Center (AMC) in Amsterdam, the Netherlands, developed the SUPPORT Coach app, the Dutch version of the U.S. PTSD Coach, which is sponsored by the Ambulance Care Netherlands. SUPPORT Coach is expected to be released to the App Store and Google Play in the spring of 2018.

The name 'SUPPORT Coach' was chosen instead of PTSD Coach based on the assumption that the app would not only be useful for individuals with PTSD, but also for those with mild and other trauma-related symptoms and that using the term PTSD could prevent some trauma survivors from using the app if they did not consider their symptoms PTSD. The content of the U.S. PTSD Coach was translated into Dutch with wording, contact addresses, and information being modified to make SUPPORT Coach suitable for a more generic trauma-exposed population. The overall look and feel is bright and clear, using simple icons, and the Information and Self-test sections conform to
DSM-5. Capitalizing on enhancements originally included in PTSD Coach Australia, the Manage Symptoms section is divided into two subsections, Tools and Symptoms. SUPPORT Coach contains a Calendar feature where users can schedule self-tests, manage exercises, and appointments and activities (see Figure 4).

Initial research on SUPPORT Coach evaluated its effectiveness as a stand-alone self-help tool for traumaexposed Dutch health care professionals (e.g., ambulance workers and hospital employees; $\mathrm{n}=287$ ) with mild or worse PTSD symptoms (i.e., primary care PTSD screen for DSM-5 $\geq 1$ ). Participants were randomized to one month of access to SUPPORT Coach plus care as usual or care as usual only. Self-report assessments of PTSD symptoms, negative trauma-related cognitions, psychological resilience, and social support were completed at baseline, posttreatment, and one-month follow-up. Results showed that $89 \%$ of the participants in the SUPPORT Coach condition downloaded the app and logged in. Promising preliminary results were found on improvements in negative traumarelated cognitions and psychological resilience, both crucial mechanisms in successfully coping with adversities (43).

Future studies should examine the potential preventive effect of SUPPORT Coach use directly after trauma, and its effectiveness among different populations with various levels of PTSD symptoms. The AMC is currently investigating the feasibility and effectiveness of the app as an adjunct to trauma-focused psychotherapy among PTSD patients.

\section{CoachPTBS Germany}

The 12-month prevalence of PTSD in the German population is estimated to be $2.3 \%$ (44) and among returning German soldiers from ISAF (International Security Assistance Force) in 2009 it was 2.9\% (45). Only about half of those soldiers with PTSD sought help (45). Smartphone ownership in Germany is estimated to be $67.3 \%$ of the population in 2017 (46). There was a chance to act upon those numbers: To overcome stigma in reaching out for help with PTSD as well as educate combat personnel and their relatives about PTSD, it was decided to build upon the success of the U.S. PTSD Coach and its spin-offs. Thus, a German version, CoachPTBS (PTBS, Posttraumatische Belastungsstörung, the German equivalent for PTSD), was released in the App Store and Google Play in July 2016, at a gathering with the German Minister of Defense. One and a half years after its release 


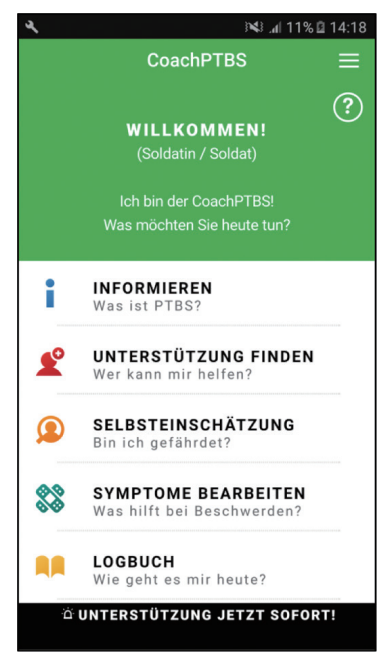

Figure 5 Home screen of CoachPTBS Germany.

it has been downloaded over $7 \mathrm{~K}$ times, with about $4 \mathrm{~K}$ of them for Android devices.

Together with the Universität der Bundeswehr in Munich (UniBwM), the Technische Universität Dresden (TUD) received a grant from the German Defense Forces and eventually received approval to begin construction of the CoachPTBS in April 2015. Many challenges in the adaption process were overcome thanks to the UniBwM's cooperation partners, the CODE Research Institute for Cyber Defense. Having expertise from these internal contractors on the team was advantageous as it was quickly decided that CoachPTBS would be reprogrammed completely.

First, upon review of the existing code, uncommented sections and fragmentations were discovered. Second, PTSD Coach was released after months of development in 2011, but innovations in app creation tend to evolve rapidly with a variety of new construction methods having since become available. Third, a clean start turned out to be more convenient as it enabled an alternative programming environment (the Ionic framework, based on Angular and Apache Cordova) that, in turn, allowed for a good division of labor between the two associated universities and eventually led to a hybrid app and thus an easier migration to the targeted operating systems (i.e., iOS and Android).

The German app retained PTSD Coach's original four main sections and added a fifth category named "Logbook" (see Figure 5) that offers a momentary assessment category featuring a diary, a quick check function, a "Worry Diary", and a daily résumé. The quick check allows users to assess their momentary emotional state in just a few seconds and monitor it over time. The worry diary helps users to gain control of troublesome thoughts. The daily résumé encourages users to reflect on positive events during the day by answering three questions, such as "What went well today?" Since the CoachPTBS exclusively works offline, information obtained this way remains entirely under the user's control. If desired, this information can be shared with a counselor to help inform treatment (e.g., for detecting behavioral patterns).

Much of CoachPTBS's content was derived from its predecessors. The U.S. and Australian versions had been carefully translated and interpreted to not only match the target language but also German culture. The creators worked closely with the German Armed Forces Center for Military Mental Health (Psychotraumazentrum), a military hospital in Berlin, as well as the Ministry of Defense for approval of the content. Additional information was also included, since, for instance, the German defense system differs greatly in some respects from the U.S.'s. Special focus was given to sleep disturbances accompanying trauma with certain parts of the CBT-I Coach app (47) being included in CoachPTBS as well.

CoachPTBS received a major update in May 2017 on its layout as well as in two key aspects: The content branched out to other disorders possibly arising after trauma, so the chapters on depression and anxiety in the "Inform" category were expanded upon. This was accompanied by adding PHQ-D items $(48,49)$ to the Self-Assessment section so users could also assess their mood and anxiety symptoms. The other major focus of this upgrade was the addition of more material for relatives of soldiers. Thus, a new category, "family and social issues" was created, drawing inspiration from the PTSD Family Coach app (50).

While usability studies of CoachPTBS occurred during the beta testing phases to identify bugs and acquire input from testers, more substantial studies on individuals who either treat or have PTSD themselves are now just beginning. For example, by consulting patients and therapists, a study on usability will keep requirements for patient-friendly apps up-to-date. A second study has been proposed that pits the CoachPTBS against participants' usual means of coping during their everyday lives (e.g., humor, optimism, or alcohol use), and includes ecological momentary assessment and a measure of hair cortisol, a biological marker of stress (51). This RCT will evaluate the benefits CoachPTBS may provide to trauma survivors 


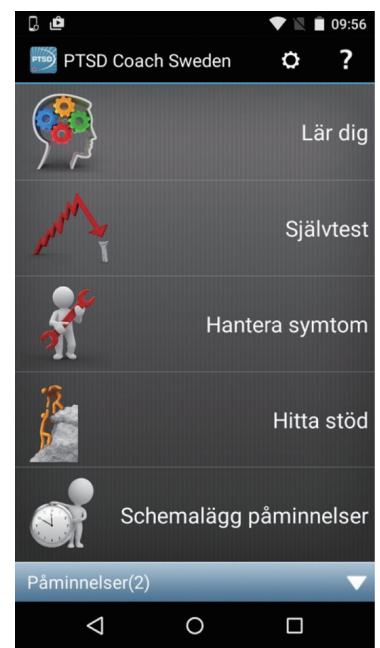

Figure 6 Home screen of PTSD Coach Sweden.

in terms of enhancing coping mechanisms and reducing PTSD and other symptoms.

\section{PTSD Coach Sweden}

The lifetime prevalence of PTSD in Sweden has been estimated to be $5.6 \%$ (52). As of 2017, 85\% of the Swedish population had a smartphone (53). The Swedish version of PTSD Coach is being developed by the National Centre for Disaster Psychiatry at the Department of Neuroscience, Uppsala University with support from The Swedish Board of Health and Welfare and The Swedish Crime Victim Compensation and Support Authority. It is intended to be of use to trauma survivors in the general population, military personnel (current and past), and as an adjunctive service to other interventions deployed after disasters.

The broader app program includes (I) app development/ adaptation; (II) pilot testing in an uncontrolled feasibility trial; (III) app updating and further adaptations; and (IV) evaluation of efficacy in an RCT. To date, PTSD Coach Sweden has been compiled in a first version (see Figure 6) available for beta-testing for both iOS and Android operating systems. The results of the pilot study have been submitted for publication and preparations for an RCT are underway. The app is planned to be released in the app stores after the RCT has ended. Uppsala University will be the publisher of the app, securing wide-scale access at no cost for users.

Initially, it was decided that it would be more effective to translate and adapt the existing U.S. version rather than to develop a similar app from scratch. In the adaptation, all text in the user interface was translated to Swedish. PTSD Coach Australia's feature allowing options for the user to select Soldier or Veteran was changed to Civilian or Military. New audio tracks were recorded in Swedish for the coping tool visualizations (e.g., progressive muscle relaxation tool), which was particularly challenging to synchronize the timings of the exercises. Contact information for organizations available for support were replaced with those in Sweden, with respect to users of both civilian and military backgrounds, and an attempt was made to include a comprehensive list of organizations. During the adaptation process, despite the original code being extensible, it was found that not all text content displayed to users was stored in separate locations with some being intertwined with the main code, which made the translation approach more demanding.

Preliminary findings from a small, pre-post pilot study of PTSD Coach Sweden that involved 11 adult participants from the general community suggest that one month of use is associated with reductions in posttraumatic stress and depressive symptoms that are similar to those found with the original version $(25,26)$. Participants appreciated the app, although their ratings of its perceived helpfulness were somewhat lower as compared to those found in the original study (14). This may be the result of the project encountering a long delay between participant recruitment and app testing, due to difficulties working with external contractors who were responsible for programming the app.

\section{PTSD Coach Denmark}

According to the Danish Veterans Center, there are about 60,000 veterans in Denmark with the majority having been deployed after 1992 (54). It is estimated that about 9\% of these veterans experience an elevated level of PTSD symptoms after two and a half years. The total number of individuals suffering from PTSD in Denmark is estimated to be 57,000 or roughly $1 \%$ of the population. As the clear majority of Danish veterans deployed since 1992 from the former Yugoslavia, Iraq, and Afghanistan are under the age of 50, it is a good guess that nearly all of them own a smartphone since more than $90 \%$ of Danes are smartphone owners (55). After overcoming technical challenges, PTSD Coach Denmark was launched simultaneously for iOS and Android in January 2017. Due to a minor technical problem, the Android version was re-launched in August 2017. It is now available for free download from the App 


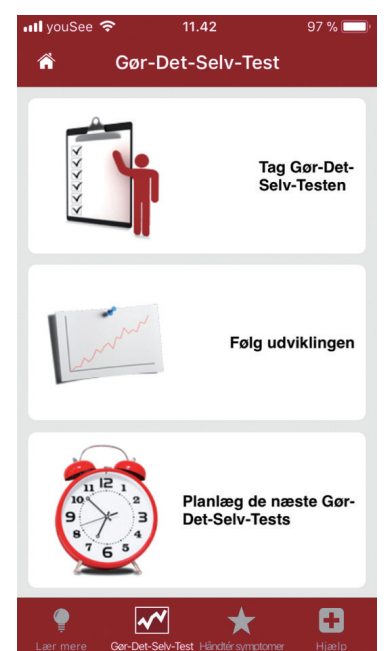

Figure 7 Track symptoms screen of PTSD Coach Denmark.

Store and Google Play. Since it was released, there have been more than $1 \mathrm{~K}$ downloads ( $75 \%$ Android) with over 300 active users within the last 30 days.

PTSD Coach Denmark was developed by Thomas Iversen, MSc, who is an Associate Professor in the Department of Psychology at Aarhus University. It is intended primarily for Danish veterans, but also for other groups suffering from PTSD. The development started in 2014 with support from the Danish Foundation (TrygFonden). While work on PTSD Coach Denmark was underway, a Danish version of the U.S. PTSD Family Coach app was started by the Danish Veterans Center, and an exchange of ideas was agreed upon. The content of the U.S. PTSD Coach was translated into Danish and modified to fit into the Danish health care system, and institutions and telephone numbers, websites and other resources were revised. Furthermore, the assessment section (see Figure 7) was updated to the PCL-5. Apart from these necessary modifications, the app was also made more explicitly inclusive for individuals exposed to other types of trauma, for instance violence, sexual assault, and road traffic accidents.

A systematic evaluation of PTSD Coach Denmark has not yet been carried out, but positive anecdotal feedback has been received from veterans, on social media, and on TV. Soon, efforts are planned to make the app known to more veterans and other individuals with PTSD. This includes public relations campaigns on various media, including social media. Recently there has been a contact from the Danish Ministry of Justice, which has shown an interest in developing a special version of the app for police officers and prison staff.

\section{Discussion}

Since PTSD Coach was released in 2011, its source code and content have been shared with individuals in six other countries around the world who have initiated building versions of PTSD Coach specifically tailored to their unique populations. To date, four new versions have been released (Australia, Canada, Germany, and Denmark) and are available for free download and the two others (Netherlands and Sweden) are slated to be released soon. This emerging global PTSD Coach community is reaping benefits of a reciprocal sharing model. Most notably, as teams iterate on PTSD Coach by including additional features (e.g., a scheduler feature in PTSD Coach Australia, Logbook in CoachPTBS Germany), improved functionality (e.g., direct access to specific self-management tools in PTSD Coach Australia), tailoring for specific populations (PTSD Coach Australia), expanded content for other groups and issues (depression, anxiety, OSI), updated code, and refined extensibility (e.g., PTSD Coach Sweden) other teams can capitalize on these enhancements. An unanticipated upshot of sharing PTSD Coach has been other apps in VA's portfolio, including PTSD Family Coach (Denmark and Germany) and CBT-I Coach (Germany) being considered for versioning or some of their content being incorporated into PTSD Coach versions.

While here we have focused on versions of PTSD Coach developed in other countries, the open sharing of code is also springing additional U.S. adaptations of PTSD Coach for other highly stressful events and traumaexposed populations, further expanding the PTSD Coach community. Among these spin-offs is Cancer Distress Coach, designed for cancer survivors struggling with the psychological aftermath of the disease and its treatment (56). Preliminary research on Cancer Distress Coach has shown promise with nearly a third (32.3\%) of cancer survivors reporting significant PTSD symptoms $(n=31)$ showing clinically meaningful symptom improvement after only a month of use. Work is also planned for a version of PTSD Coach for police officers, a group who are especially resistant to acknowledging PTSD symptoms and seeking care for them (57). As was mentioned above, similar efforts are being considered for an adaptation of PTSD Coach Denmark targeting Danish police and prison staff.

Notwithstanding this success, versioning PTSD Coach 
is not without challenges. Foremost among these is the significant resources required to build an app especially if separate native iOS and Android apps are desired. While there are ways to increase efficiency and potentially reduce expenses, as the German team demonstrated with building a hybrid app, substantial costs are still involved. Importantly, it should be noted that maintaining and updating apps (e.g., to remain compatible with operating system updates) also involves significant resources. Other challenges include often having to work with external contractors who have the requisite app coding expertise (e.g., PTSD Coach Sweden). Issues can stem from collaborating across disciplines where conflicts can arise due to members speaking different professional languages and having different expectations (e.g., timeframes, division of labor, deliverables) (11). Finally, translating apps into other languages can create formatting and presentation problems, as was experienced by the developers of PTSD Coach Sweden with synchronizing audio recording and visual presentations.

Aside from these challenges versioning an app, mobile apps themselves have shortcomings that must also be acknowledged. Most obvious among these is that they require smartphones or other mobile devices for use. These devices can be cost prohibitive for many individuals, especially those in economically depressed areas and developing countries. Thus, it is no surprise that all seven countries with versions of PTSD Coach are developed, western nations with high smartphone penetration. In fact, smartphone uptake across and within regions and markets is not balanced, leaving behind many who could benefit from them. Eastern Africa and South Asia are regions most lagging, with smartphone ownership at $25 \%$ and $30 \%$ respectively-much lower than the global average of over $50 \%(58)$.

Given these challenges, other possible delivery platforms and models of adapting for additional populations may need to be considered, including building web-based versions. Based on requests from veterans and members of the public without smartphones, the National Center for PTSD created a web-based version of PTSD Coach called PTSD Coach Online (https://www.ptsd.va.gov/apps/ ptsdcoachonline/default.htm) to provide access to them. A related option is a mobile-enabled website (one with responsive web design that works equally well on desktop computers to mobile devices). Such sites are advantageous because they can be used by anyone with access to the internet with a web browser and can even be designed to appear as an app on mobile devices. When considering these options, their limitations should be weighed as well, including the requirement of a reliable Internet connection for use and an inability to take advantage of existing smartphone features (e.g., contacts, calendar, phone).

Like other e-health options, availability and use of apps could be limited when infrastructure is compromised, for example during natural disasters (e.g., hurricane Maria in Puerto Rico), civil unrest, and military conflicts, when they are most needed. PTSD Coach was intentionally designed to work offline and require minimal bandwidth for downloading, which could mitigate the impact of such disruptions. Finally, mobile apps cannot increase problem recognition if they are not downloaded and used. Therefore, a concerted effort involving a public health education campaign about trauma and PTSD along with promotion of the app may be required, possibly even encouraging download prior to a foreseeable disaster. PTSD Coach was intended to be a first care step possibly reducing an individual's symptoms and the overall public health impact of trauma if received shortly after one (59). The intriguing findings from The Netherlands showing that SUPPORT Coach could reduce negative cognitions and enhance resilience (43) are encouraging as these factors are important in recovery after trauma. Despite this, PTSD Coach will not be the complete or only solution for many trauma survivors; therefore, additional care resources will still be required, which, as has been mentioned, may not be available.

How helpful these newest versions of PTSD will be remains to be seen. It is encouraging that many of these versions are or will be undergoing their own research evaluations. The original version has already distinguished itself from the vast majority of mental health-related apps that populate the app stores (60) as it has already shown promise in several RCTs. Despite this, more research is needed, including cost effectiveness research and studies with active comparators. In addition, mobile apps such as the PTSD Coach open interesting research possibilities to better understand how symptoms fluctuate over the course of people's daily lives and how the use of specific types of content influence the user's particular problems.

\section{Conclusions}

The increasing ubiquity of smartphones worldwide offers a novel opportunity to reach and provide lowintensity interventions at scale to individuals suffering the psychological aftermath of exposure to traumatic 
events. Given the intractable problems with traditional mental health care (e.g., limited availability of providers), smartphone apps such as PTSD Coach could assist in addressing the enormous global unmet need for care. Seizing on this opportunity, the six additional versions of PTSD Coach that have been or are being developed around the world through a model of open sharing of app code and content represent an encouraging start. However, much work remains to ensure that apps like PTSD Coach are available to those in areas where need is greatest and resources are scant or nonexistent. Fortunately, as technology advances, the cost of smartphones and app development should come down, which will afford greater reach and impact of this novel way of intervening. Technology advances may also provide innovative opportunities that could increase the power of mobile interventions (e.g., through information gathered from wearable sensors and other available data streams) that could deliver more timely intervention as well as an improved understanding of posttraumatic reactions themselves.

\section{Acknowledgements}

None.

\section{Footnote}

Conflicts of Interest: This manuscript is partially based on: PTSD Coach around the World: A Global Perspective on a Smartphone App Designed for Self-management of PTSD Symptoms. Symposium presented at the annual meeting of the International Society for Traumatic Stress Studies, Dallas, TX, November, 2016. Chair: Christianne van der Meer.

\section{References}

1. Kessler RC, Sonnega A, Bromet E, et al. Posttraumatic Stress Disorder in the National Comorbidity Survey. Arch Gen Psychiatry 1995;52:1048.

2. Koenen KC, Ratanatharathorn A, Ng L, et al. Posttraumatic stress disorder in the World Mental Health Surveys. Psychol Med 2017;47:2260-74.

3. Diagnostic and statistical manual of mental disorders. 5th ed. Arlington, VA: American Psychiatric Association, 2013.

4. Brancu M, Mann-Wrobel M, Beckham J, et al. Subthreshold posttraumatic stress disorder: A metaanalytic review of DSM-IV prevalence and a proposed
DSM-5 approach to measurement. Psychol Trauma 2016;8:222-32.

5. Harik JM, Matteo R, Hermann B, et al. What people with PTSD symptoms do (and do not) know about PTSD: A national survey. Depression Anxiety 2017;34:374-82.

6. Kantor V, Knefel M, Lueger-Schuster B. Perceived barriers and facilitators of mental health service utilization in adult trauma survivors: A systematic review. Clin Psychol Rev 2017;52:52-68.

7. Kazdin AE. Addressing the treatment gap: A key challenge for extending evidence-based psychosocial interventions. Behav Res Ther 2017;88:7-18.

8. Kuester A, Niemeyer H, Knaevelsrud C. Internet-based interventions for posttraumatic stress: A meta-analysis of randomized controlled trials. Clin Psychol Rev 2016;43:1-16.

9. Van Ameringen $M$, Khalesi Z, Pullia K, et al. There is an app for that! The current state of mobile applications (apps) for DSM-5 obsessive-compulsive disorder, posttraumatic stress disorder, anxiety and mood disorders. Depression Anxiety 2017;34:526-39.

10. Number of smartphone users worldwide from 2014 to 2020 (in billions). Statista 2015 [cited 12 April 2018]. Available online: https://www.statista.com/statistics/330695/ number-of-smartphone-users-worldwide/

11. Olff $M$. Mobile mental health: a challenging research agenda. Eur J Psychotraumatol 2015;6:27882.

12. Price M, Yuen E, Goetter E, et al. mHealth: A Mechanism to Deliver More Accessible, More Effective Mental Health Care. Clin Psychol Psychother 2014;21:427-36.

13. Hoffman JE, Wald LJ, Kuhn E, et al. PTSD Coach. iTunes, 2017. Available online: http://itunes.apple.com

14. Kuhn E, Greene C, Hoffman J, et al. Preliminary Evaluation of PTSD Coach, a Smartphone App for PostTraumatic Stress Symptoms. Mil Med 2014;179:12-8.

15. Kessler RC, Berglund P, Demler O, et al. Lifetime Prevalence and Age-of-Onset Distributions of DSMIV Disorders in the National Comorbidity Survey Replication. Arch Gen Psychiatry 2005;62:593.

16. Kulka R, Schlenger W, Fairbank J, et al. Trauma and the Vietnam war generation: report of findings from the national Vietnam veterans readjustment study. New York: Brunner/Mazel; 1990.

17. Kang HK, Natelson B, Mahan C, et al. Post-Traumatic Stress Disorder and Chronic Fatigue Syndrome-like Illness among Gulf War Veterans: A Population-based Survey of 30,000 Veterans. Am J Epidemiol 2003;157:141-8.

18. Jaycox L, Tanielian T. Invisible wounds of war. Santa 
Monica, CA: RAND Center for Military Health Policy Research, 2008.

19. Mobile Fact Sheet. Pew Research Center: Internet, Science \& Tech 2018 [cited 12 April 2018]. Available online: http://www.pewinternet.org/fact-sheet/mobile/

20. Kimball R. Genachowski and Copps Present AAA Awards. Federal Communications Commission 2018 [cited 12 April 2018]. Available online: http://www.fcc.gov/document/ genachowski-and-copps-present-aaa-awards

21. American Telemedicine Association. ATA Award Winners and New Fellows Represent Leaders in Telemedicine, mHealth. Prnewswire.com 2018 [cited 24 May 2013]. Available online: http://www.prnewswire.com/newsreleases/ata-award-winners-and-new-fellows-representleaders-in-telemedicine-mhealth-146820315.html

22. Ruzek JI, Hoffman J, Ciulla R, et al. Bringing Internetbased education and intervention into mental health practice: afterdeployment.org. Eur J Psychotraumatol 2011;2:7278.

23. Foa E. Effective treatments for PTSD 2nd ed. New York, NY: Guilford Press; 2009.

24. Weathers F, Litz B, Keane T, et al. PTSD Checklist for DSM-5 (PCL-5) - PTSD: National Center for PTSD. Ptsd.va.gov 2018 [cited 24 May 2013]. Available online: https://www.ptsd.va.gov/professional/assessment/adult-sr/ ptsd-checklist.asp

25. Miner A, Kuhn E, Hoffman J, et al. Feasibility, acceptability, and potential efficacy of the PTSD Coach app: A pilot randomized controlled trial with community trauma survivors. Psychol Trauma 2016;8:384-92.

26. Kuhn E, Hoffman J, Garvert D, et al. A randomized controlled trial of a smartphone app for posttraumatic stress disorder symptoms. J Consult Clin Psychol 2017;85:267-73.

27. Keen S, Roberts N. Preliminary evidence for the use and efficacy of mobile health applications in managing posttraumatic stress disorder symptoms. Health Systems 2017;6:122-9.

28. Owen JE, Jaworski B, Kuhn E, et al. mHealth in the Wild: Using Novel Data to Examine the Reach, Use, and Impact of PTSD Coach. JMIR Mental Health 2015;2:e7.

29. Possemato K, Kuhn E, Johnson E, et al. Using PTSD Coach in primary care with and without clinician support: a pilot randomized controlled trial. Gen Hosp Psychiatry 2016;38:94-8.

30. Hoffman JE, Ramsey KM, Estrin D. Open architecture and standards in mobile health. In: Marsch LA, Lord SE, Dallery J. editors. Behavioral Healthcare and Technology:
Using Science-Based Innovations to Transform Practice. Oxford University Press, 2014:264-80.

31. Center for Posttraumatic Mental Health. Trauma Research at Phoenix Australia. Phoenix Australia. 2018 [cited 12 April 2018]. Available online: http://phoenixaustralia.org/ resources/trauma-research/

32. Mobile Consumer Survey 2017 | Deloitte Australia | Technology, Media \& Telecommunications, Mobile, Trends 2018 [cited 12 April 2018]. Available online: https://www2.deloitte.com/au/mobile-consumer-survey

33. McFarlane AC, Hodson SE, Van Hooff M, et al. Mental health in the Australian Defence Force: 2010 ADF Mental Health and Wellbeing Study: Full report. Department of Defence: Canberra, 2011.

34. Australia Department of Veterans Affairs and Department of Defence. PTSD Coach Australia. Canberra; 2018 [cited 12 April 2018]. Available online: http://at-ease.dva.gov. au/veterans/files/2013/01/Clinicians_Guide_to_PTSD_ Coach_Australia2.pdf

35. Langlois K, Samokhvalov A, Rehm J, et al. Health state descriptions for Canadians. Statcan.gc.ca 2018 [cited 12 April 2018]. Available online: http://statcan.gc.ca/pub/82619-m/82-619-m2012004-eng.htm

36. Pearson C, Zamorski M, Janz T. Mental health of the Canadian Armed Forces. Statcan.gc.ca 2018 [cited 12 April 2018]. Available online: http://statcan.gc.ca/pub/82624-x/2014001/article/14121-eng.htm

37. Smartphone Behaviour in Canada and the Implications for Marketers in 2016 - Catalyst. Catalyst 2018 [cited 12 April 2018]. Available online: http://catalyst.ca/2016-canadiansmartphone-behaviour/

38. Canada V. Minister Blaney Announces National Launch of PTSD Mobile App and funding for PTSD Research Canada.ca. Canada.ca 2018 [cited 12 April 2018]. Available online: https://www.canada.ca/en/news/archive/2013/05/ minister-blaney-announces-national-launch-ptsd-mobileapp-funding-ptsd-research.html

39. Research and information - Mental Health - Health and well being - Services - Veterans Affairs Canada. Veterans. gc.ca 2018 [cited 12 April 2018]. Available online: http:// www.veterans.gc.ca/eng/services/health/mental-health/ understanding-mental-health

40. Current Issues in Mental Health in Canada: Mental Health in the Canadian Forces and Among Veterans (in Brief). Ottawa: Library of Parliament; 2018 [cited 12 April 2018]. Available online: https://lop.parl.ca/Content/LOP/ ResearchPublications/2013-91-e.pdf

41. de Vries GJ, Olff M. The lifetime prevalence of 
traumatic events and posttraumatic stress disorder in the Netherlands. J Trauma Stress 2009;22:259-67.

42. Moeskops M. Markt mobiele devices volledig verzadigd. Gfk.com 2017 [cited 12 April 2018]. Available online: http://www.gfk.com/nl/insights/press-release/marktmobiele-devices-volledig-verzadigd/

43. Bakker C, Holmersma A, Buschbach S, et al. Effectiveness of the Dutch PTSD Coach to Reduce Posttraumatic Stress Symptoms in Trauma-exposed Health Care Professionals. Presentation presented at International Society for Traumatic Stress Studies, 2018.

44. Jacobi F, Siegert M, Mack S, et al. Twelve-month prevalence, comorbidity and correlates of mental disorders in Germany: the Mental Health Module of the German Health Interview and Examination Survey for Adults (DEGS1-MH). Int J Methods Psychiatr Res 2014;23:304-19.

45. Wittchen HU, Schonfeld S, Kirschbaum C, et al. Traumatic experiences and posttraumatic stress disorder in soldiers following deployment abroad: how big is the hidden problem?. Dtsch Arztebl Int 2012;109:559-68.

46. Statista. Smartphones - Penetration in Europa nach Land bis 2021 I Prognose. Statista 2018 [cited 12 April 2018]. Available from: https://de.statista.com/statistik/ daten/studie/321967/umfrage/prognose-zum-anteil-dersmartphone-nutzer-in-europa-nach-land/

47. Kuhn E, Weiss B, Taylor K, et al. CBT-I Coach: A Description and Clinician Perceptions of a Mobile App for Cognitive Behavioral Therapy for Insomnia. J Clin Sleep Med 2016;12:597-606.

48. Lowe B. UniversitätsKlinikum Heidelberg: Startseite. Klinikum.uni-heidelberg.de 2001 [cited 12 April 2018]. Available from: https://www.klinikum.uni-heidelberg. de/...PHQ_Manual1.pdf

49. Spitzer RL, Kroenke K, Williams J, et al. Validation and utility of a self-report version of PRIME-MD: the PHQ primary care study. JAMA 1999;282:1737-44.

50. Owen JE, Jaworski BK, Kuhn E, et al. Development of a mobile app for family members of Veterans with PTSD: identifying needs and modifiable factors associated with burden, depression, and anxiety. J Fam Stud 2017:1-22.

51. Stalder T, Kirschbaum C. Analysis of cortisol in hair State of the art and future directions. Brain Behav Immun 2012;26:1019-29.

52. Frans O, Rimmo P, Aberg L, et al. Trauma exposure and post-traumatic stress disorder in the general population. Acta Psychiatrica Scandinavica 2005;111:291-9.

53. Swedes and the Internet 2017. Soi2017.se 2017 [cited 12 April 2018]. Available online: http://www.soi2017.se/

54. Veterancentret - Research database. Pure.fak.dk 2018 [cited 12 April 2018]. Available online: http://pure.fak.dk/ portal/en/organisations/veterancentret(245721fd-54e04fe5-b7df-87d12e9a9ffc).html

55. Danish Media Research, Kantar Gallup. Globalisation of the Danish Media Industry. Slks.dk 2017 [cited 12 April 2018]. Available online: https://slks.dk/fileadmin/user_ upload/dokumenter/medier/Mediernes_udvikling/2017/ Specialrapporter/Internationale_aktoerer/Rapport_og bilag/Summary_in_English.pdf

56. Smith SK, Kuhn E, O'Donnell J, et al. Cancer distress coach: Pilot study of a mobile app for managing posttraumatic stress. Psychooncology 2018;27:350-3.

57. Karaffa K, Koch J. Stigma, Pluralistic Ignorance, and Attitudes Toward Seeking Mental Health Services Among Police Officers. Crim Justice Behav 2016;43:759-77.

58. Karlsson M, Penteniari G, Croxson H, et al. Accelerating affordable smartphone ownership in emerging markets. Walbrook: GSMA, 2018.

59. Roberts NP, Kitchiner N, Kenardy J, et al. Systematic Review and Meta-Analysis of Multiple-Session Early Interventions Following Traumatic Events. Am J Psychiatry 2009;166:293-301.

60. Bakker D, Kazantzis N, Rickwood D, et al. Mental Health Smartphone Apps: Review and Evidence-Based Recommendations for Future Developments. JMIR Mental Health 2016;3:e7. doi: $10.21037 /$ mhealth.2018.05.01

Cite this article as: Kuhn E, van der Meer C, Owen JE, Hoffman JE, Cash R, Carrese P, Olff M, Bakker A, Schellong J, Lorenz P, Schopp M, Rau H, Weidner K, Arnberg FK, Cernva M, Iversen T. PTSD Coach around the world. mHealth 2018;4:15. 